Boletín de la Sociedad Geológica Mexicana

Número Especial de Geología Urbana

Toмo LVIII, NÚM. 2, 2006, P. 253-257

NOTA TÉCNICA

\title{
Análisis de la actividad sísmica en el Volcán Popocatépetl (México) durante el periodo 1994-1997
}

\author{
Javier Lermo-Samaniego*, Yanet Antayhua-Vera, Marcos Chavacán-Ávila \\ Instituto de Ingeniería, Universidad Nacional Autónoma de México, Ciudad Universitaria, 04510 México, D.F. \\ *jles@pumas.iingen.unam.mx
}

\section{Resumen}

En este estudio, se realiza un análisis detallado de los sismos volcano-tectónicos registrados por la Red Sísmica instalada en el volcán Popocatepetl durante el periodo 1994-1999, a fin de conocer sus parámetros hipocentrales y la naturaleza de la fuente que los origina. La base de datos contiene 115 sismos registrados en 5 o más estaciones sísmicas. Los resultados muestran que los sismos presentan magnitudes $\mathrm{Md}<3.2$, profundidades menores a $12 \mathrm{~km}$ y errores en la localización hipocentral menores a $1 \mathrm{~km}$. Las distribuciónes epicentral e hipocentral muestran 2 agrupamientos de sismos en zonas específicas, la primera (Zona A) localizada bajo el cráter del volcán y la segunda (Zona B) al SE de la misma. El análisis de las formas de onda y mecanismos focales para ambas zonas muestra que los sismos correspondientes a la Zona A presentan fases emergentes y diferentes tipos de mecanismos focales, indicando que esta sismicidad puede estar relacionada a procesos de fracturamiento de las paredes del volcán como resultado de la ascensión del magma. Los sismos de la zona B, en cambio, presentan fases impulsivas y mecanismos focales de tipo normal con una pequeña componente de transcurrencia (lateral izquierda). Este patrón sugiere que los sismos de la Zona B podrían estar asociados a un fallamiento de tipo normal en dirección NW-SE, coincidiendo con la dirección y mecanismos focales observados en el sistema de fallas "La Pera", localizado cerca del volcán Popocatépetl.

Palabras clave: Volcán Popocatépetl, sismicidad, mecanismos focales, Sistema de Fallas La Pera.

Abstract

In this study, a detailed analysis of the volcano-tectonic earthquakes recorded by the Seismic Network installed in the volcano Popocatepetl during the period 1994-1999 was made. The objective was to determine hypocentral parameters and the nature of the involved seismic source. Results show that earthquakes have magnitudes $\mathrm{Md}<3.2$, depths lower than $12 \mathrm{~km}$ and hypocentral location errors lower than $1 \mathrm{~km}$. Epicentral and hypocentral distributions allow to distinguish two groups of earthquakes, each having an specific location: The first one (A-Zone) located under the crater and the second one, SE of the previous one. For the events in the A-zone, the analysis of waveforms and focal mechanisms shows seismic records displaying emergent arrivals and a variety of focal mechanisms, indicating that this seismicity may be related to fracture processes in the volcano walls as a result of magmatic ascension. B-zone earthquakes present impulsive arrivals and normal mechanisms with a left-lateral component of motion. This pattern suggest that these events may be associated to normal faulting in a NW-SE direction, coinciding with both the observed direction and focal mechanisms of the fault system "La Pera", located near the volcano.

Key words: Volcano Popocatépetl, seismicity, focal mechanisms, Fault System La Pera. 


\section{Introducción}

La presencia de elementos tectónicos activos tales como sistemas de fallas, volcanes y sismicidad en la Faja Volcánica Mexicana (FVM, Fig. 1a), está íntimamente relacionado con el proceso de subducción de las placas de Cocos y Rivera (Singh et al., 1993). La Cuenca de México (CM) está localizada dentro de la FVM (Fig. 1b) y rodeada por cadenas montañosas como Pachuca, Ajusco, Las Cruces, Río Frío, Chichinautzin y Nevada. Un importante sistema de fallas y vulcanismo en la $\mathrm{CM}$ es el conocido como "La Pera", orientado en dirección aproximadamente E-W (Lermo et al., 1995a,b; Lermo et al., 1997; Lermo et al., 2001; Chavacán, 2003) y en la Sierra Nevada está ubicado el volcán Popocatépetl, motivo de este estudio. Popocatépetl (5452 m.s.n.m.) es un volcán constituido mayoritariamente por andesitas localizado en la región central de la FVM, a 65 km en dirección SE de la Ciudad de México, la más poblada de la República Mexicana. Durante un periodo de unos 70 años, la actividad eruptiva del volcán Popocatépetl estuvo dominada por emisión fumarólica (Siebe et al., 1996). Sin embargo, el monitoreo sísmico del mismo se implementó en Diciembre de 1994 con la instalación de estaciones sísmicas alrededor del volcán Popocatépetl debido al incremento de la actividad sísmica observada desde Octubre del mismo año (Lermo et al., 1995b). En general, la intensa actividad sísmica del volcán inicia el 21 de Diciembre de 1994 con la ocurrencia de sismos volcano-tectónicos (VT), sismos de periodo largo (LP) y tremores (Lermo et al. 1995b; Valdés et al., 1995). En este estudio, se analiza distribución y parámetros de fuente de los sismos VT registrados durante el periodo Diciembre 1994-1999.

\section{Datos}

El monitoreo sísmico del volcán Popocatépetl inició en 1987 con la instalación de la estación Altzomoni (IIA). Posteriormente, el Instituto de Ingeniería de la UNAM (IdeI-UNAM) y el Centro Nacional de Prevención de Desastres (CENAPRED) instalaron redes sísmicas temporales y permanentes (R.S.V.P.) en Diciembre de 1994. El números de estaciones fue incrementando gradualmente hasta la actualidad (Figura 1b). La información sísmica proveniente de estas redes fue utilizado por Lermo et al. (1995b) y Valdés et al. (1996) para clasificar los sismos en tres tipos: VT, LP y Tremores. Su evolución durante el periodo de estudio es mostrado en la Figura 2a y 2b (Huidobro-González, 2000; Arciniega, 2001).

\section{Sismicidad}

Del total de sismos VT registrados por las redes sísmicas del volcán Popocatépetl, 115 han sido seleccionados por presentar tiempos de arribo de ondas $\mathrm{P}$ y S claros. La localización hipocentral de los mismos fue realizada con el programa SEISAN (Havskov y Ottemoller, 2003), el modelo de estructura fue propuesto por Valdés et al. (1995), y una relación de velocidad $\mathrm{Vp} / \mathrm{Vs}=1.76$ y la expresión para la magnitud de duración fue propuesta por Chavacán et al. (2004). La localización hipocentral de los 115 sismos VT muestran errores menores a $2.0 \mathrm{~km}$ para el periodo $1994-1995$ y de $1.0 \mathrm{~km}$ para $1996-1999$ respectivamente. La profundidad de los sismos es menor a $12 \mathrm{~km}$ y sus magnitudes se encuentran entre 1.5 y 3.2 Md. En la Figura 3a se muestra su distribución epicentral, en la que se observan claramente 2 grupos de sismos en zonas específicas. La primera (Zona A) se distribuye alrededor del volcán y la segunda (Zona $\mathrm{B}$ ) en su extremo SE, y entre ellas se observa una ausencia de sismicidad. La distribución en profundidad de los sismos es mostrada en la Figura 3b según el perfil C-C' de la Figura 3a. En esta Figura se observa que los sismos presentan profundidades focales de 3 a $8 \mathrm{~km}$ en la Zona A y de 7 a 10 $\mathrm{km}$ en la Zona B. Otros sismos muy dispersos alcanzan profundidades del orden de $12 \mathrm{~km}$. Asimismo, a fin de conocer el origen de los sismos ocurridos en ambas zonas se desarrollan y analizan mecanismos focales utilizando la polaridad de la onda $P$ fácilmente identificable y registrada en 6 o más estaciones. Los resultados muestran diversos tipos de mecanismos para la zona A y de tipo normal con pequeñas componentes de transcurrencia para la Zona B (Figura 3c).

\section{Discusión y conclusiones}

En el análisis de la distribución epicentral y en profundidad de los sismos VT ocurridos durante el periodo Diciembre 1994 - Diciembre 1999, se observan diferencias importantes tales como el agrupamiento de sismos en las zonas A y B y el rango de profundidades de los mismos (Figura 3a y 3b). Ello sugiere que los sismos en ambas zonas se originaron por fracturamiento de rocas, mientras que los esfuerzos que los producen no son los mismos. Estos resultados son presentados en la Figura 4 y en ella podemos ver claramente que la Zona A presenta mecanismos de tipo inverso, normal, de transcurrencia y normales con componentes de transcurrencia; en tanto, los mecanismos de la Zona B son de tipo normal con pequeñas componentes de transcurrencia orientados en dirección NW-SE, similares a los obtenidos por otros autores para los sismos ocurridos en el sistema de fallas "La Pera" y en otras zonas dentro de la CM (Lermo et al., 1995a,b; Lermo et al., 1997; Lermo et al., 2001, Chavacán, 2003). Por lo tanto, los sismos de la Zona B podrían haber sido originados por esfuerzos tensionales que reactivaron una falla antigua o generaron una nueva, que entró en actividad como resultado de la intensa actividad eruptiva del volcán Popocatépetl durante los últimos 10 años. 


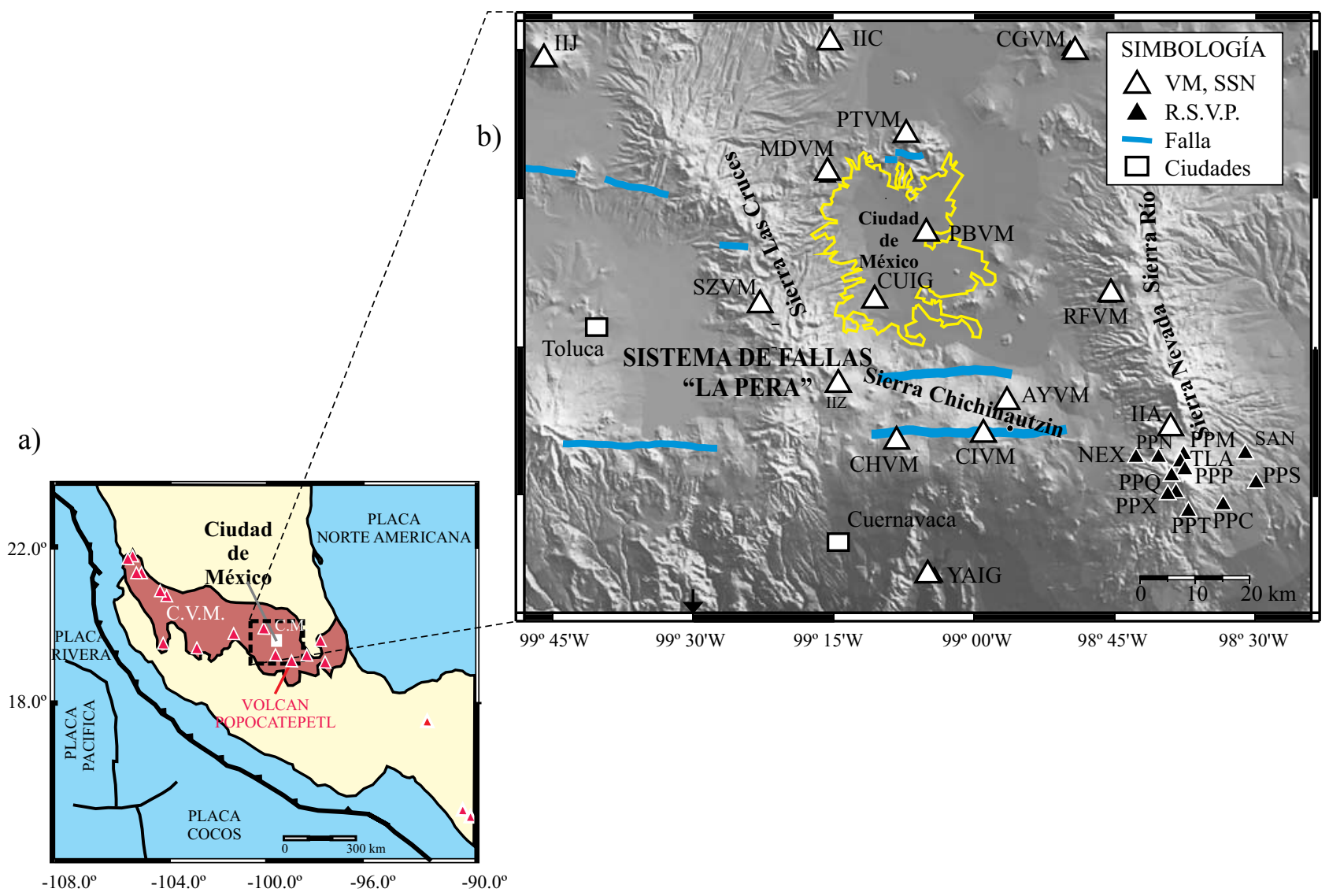

Figura 1. a) Ubicación geográfica de la Faja Volcánica Mexicana (FVM). b) La Cuenca de México (CM) y el volcán Popocatépetl.

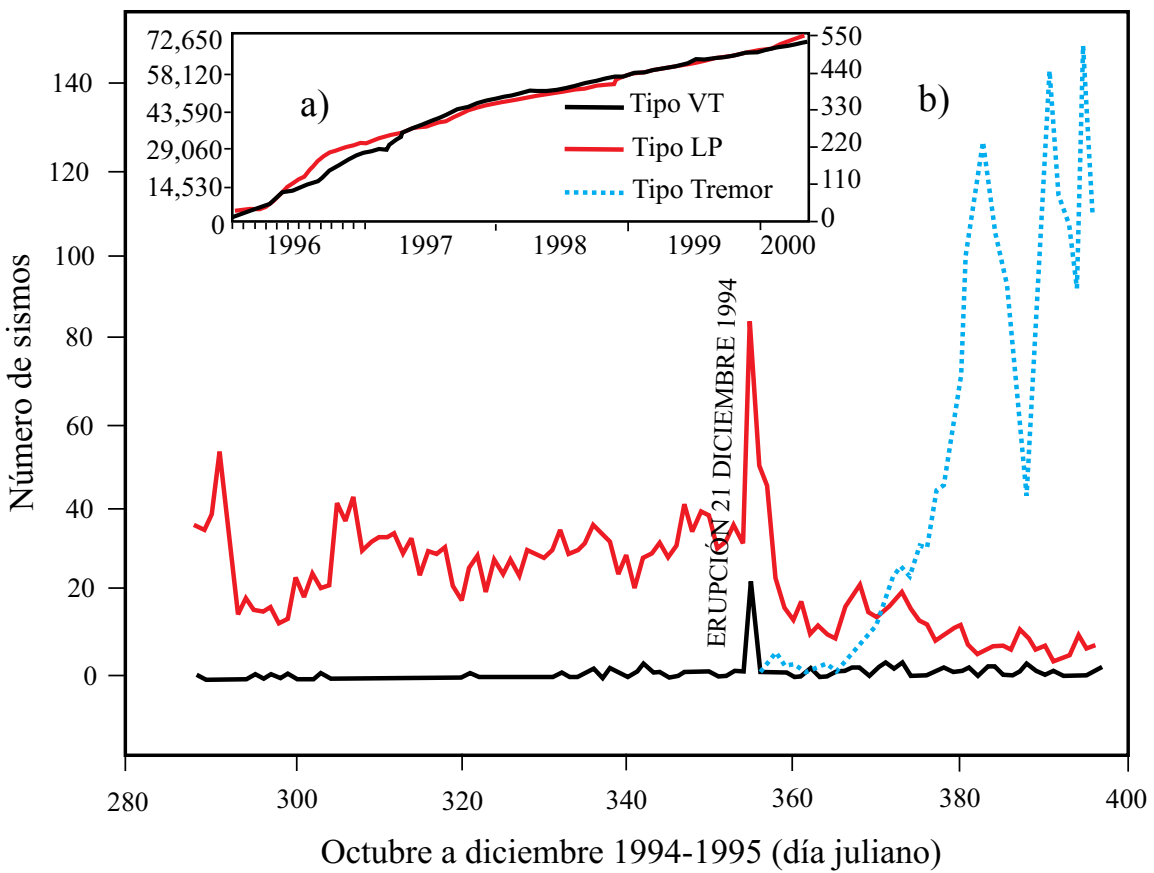

Figura 2. a) Número de sismos VT y LP por año observados por Arciniega (2001). b) Tipos de sismos observados en la estación PPX entre diciembre 1994 y 1995 (Huidobro-González, 2000). 
a)

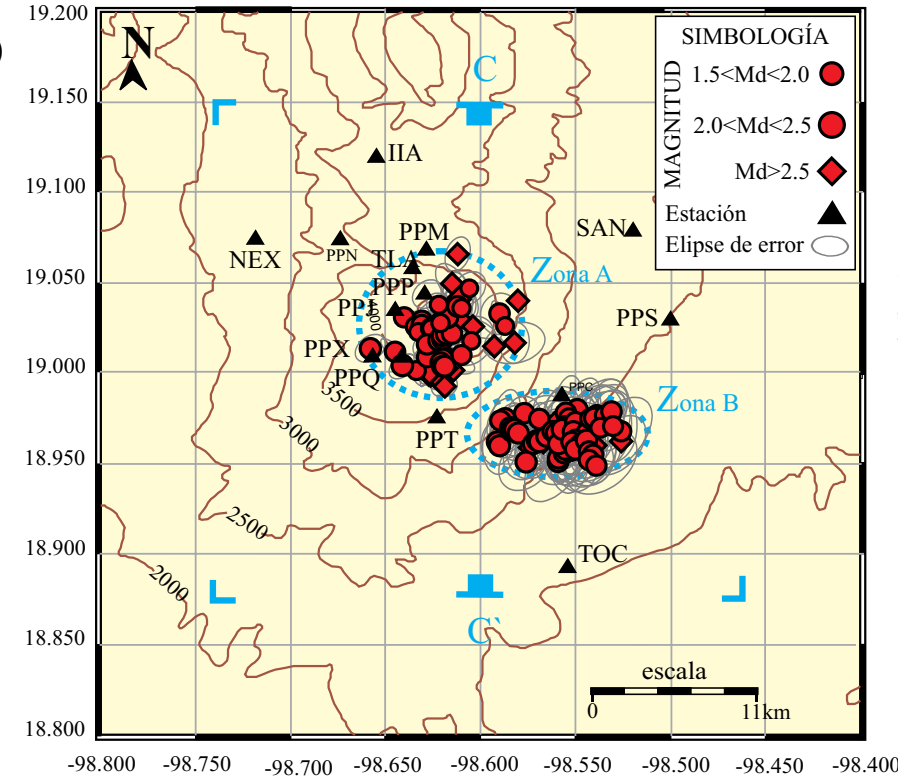

b)

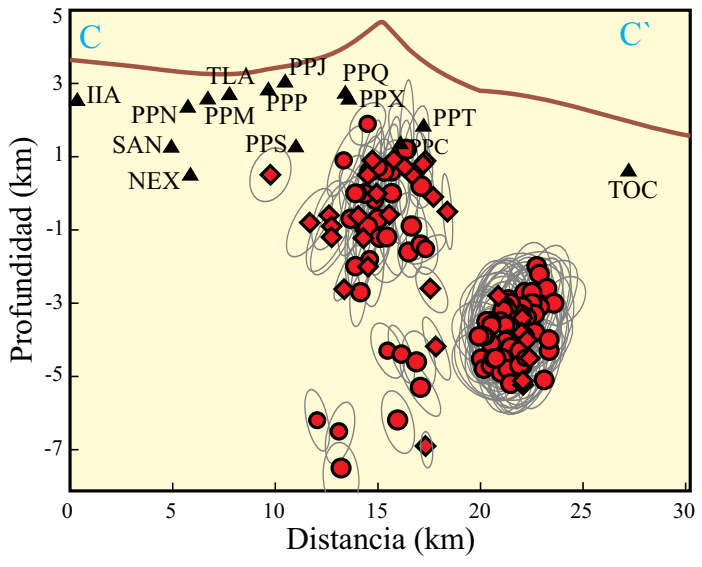

c)
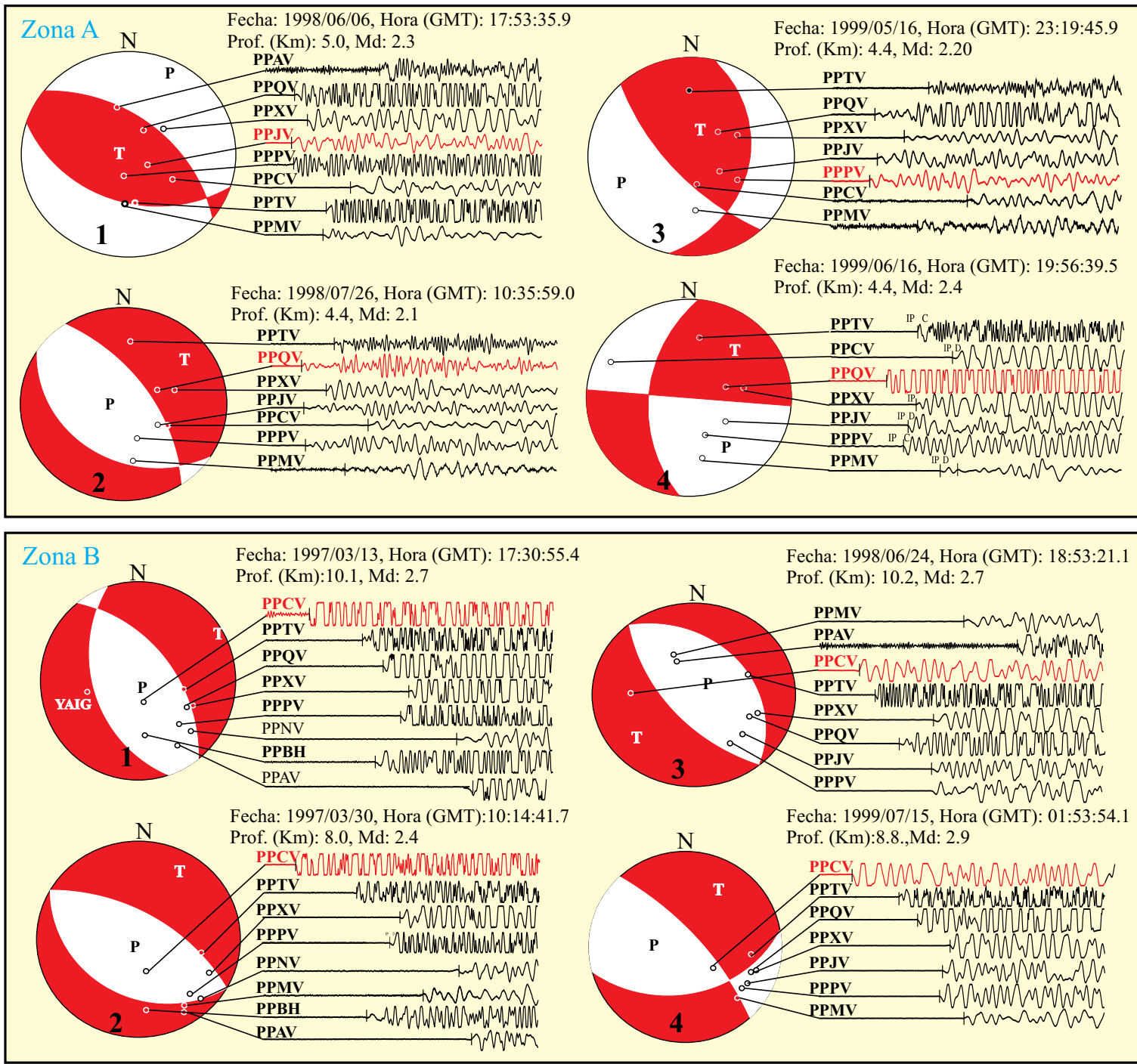

Figura 3. Distribución de los sismos ocurridos en el volcán Popocatépetl desde diciembre 1994 a 1999. a) Distribución epicentral. b) En profundidad. c) Formas de onda y solución de mecanismos focales de 4 sismos ocurridos en las zonas A y B. 


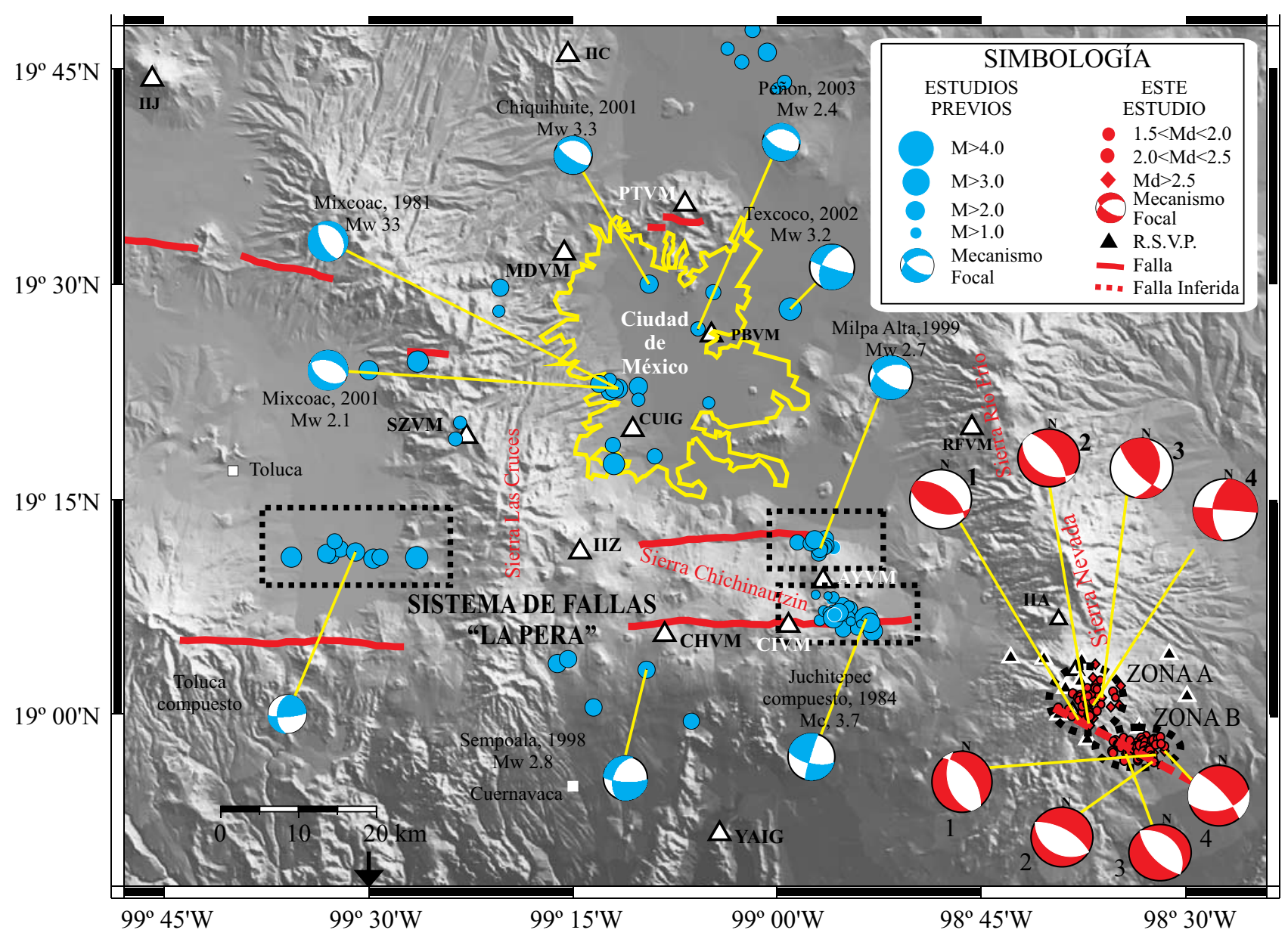

Figura 4. Distribución de la sismicidad en la CM y solución de sus mecanismos focales.

\section{Referencias bibliográficas}

Arciniega, A., 2001, Análisis de datos sísmicos de banda ancha registrados en el volcán Popocatépetl, México: Posgrado en Ciencias de la Tierra, Universidad Nacional Autónoma de México, Tesis doctoral, $192 \mathrm{p}$.

Chavacán, M., 2003, Parámetros de fuente asociados a la sismicidad del sistema de fallas de la Sierra del Chichinautzin: Facultad de Ingeniería, Universidad Nacional Autónoma de México, Tesis de Licenciatura, $90 \mathrm{p}$.

Chavacán, M., Lermo, J., Quintanar, L., 2004, Determinación de una escala de magnitud para temblores corticales en la parte central del Cinturón Volcánico Mexicano, en I Conf. Int. Peligrosidad e Ingeniería Sísmica, Universidad de Oriente, Santiago de Cuba, Cuba, CD.ROM.

Havskov, J., Ottemoller, L., 2003, SEISAN: The earthquake analysis software, version 8.0: Bergen, Norway, Department of Earth Science, University of Bergen (ed.), $250 \mathrm{p}$.

Huidobro-González, A., 2000, Actividad sísmica observada en la crisis volcánica del volcán Popocatepetl: Facultad de Ingeniería, Universidad Nacional Autónoma de México, Tesis de Licenciatura, $90 \mathrm{p}$.

Lermo-Samaniego, J., Nieto-Obregon, J., Delgado-Granados, H., Rodriguez-Gonzalez, M., Huidobro, A., Silva, G., Mendoza C., Arellano, J., 1995a, La Pera detachment fault system: active faulting south of Mexico City (I): Seismological evidence: GEOS, 15, 67.

Lermo, J., Cuenca, J., Chávez-García, F.J., Huidobro, A., Bermúdez, M.L., Rodríguez, M., Quaas, R., Sanchez-Sesma, F.J., Nava, E., Mijares, H., Briones, E., 1995b, Características espectrales de temblores Tipo-A,
Tipo-B y tremores asociados a la erupción del Volcán Popocatépetl, México, en Diciembre de 1994, en CENAPRED-UNAM (ed.), Volcán Popocatépetl, Estudios realizados durante la crisis de 1994-1995: México, D.F., 139-166.

Lermo J., Soto, J., Huidobro, A., Cuenca, J., Mijares, H., Meza, C., Flores, J., 1997, Análisis preliminar de la sismicidad en la cuenca de México y zonas aledañas, en XI Congreso Nacional de Ingeniería Sísmica, 205-215.

Lermo, J., Havskov, J., Soto, J., 2001, Sistema de información sismotelemétrica de México (SISMEX). Veintisiete años de servicio, en XIII Congreso Nacional de Ingeniería Sísmica: Guadalajara, Jal. México, Sociedad Mexicana de Ingeniería Sísmica, artículo T11A06, $17 \mathrm{p}$.

Siebe, C., Abrams, M., Macias, J.L., Obenholzner, J., 1996, Repeated volcanic disasters in Prehispanic time at Popocatépetl, central México; Past key to the future ?: Geology, 24, 399-402.

Singh S.K., Pardo, M., 1993, Geometry of the Benioff Zone and state of stress in the overriding plate in Central Mexico: Geophysical Research Letters, 20, 1483-1486.

Valdés, C., González, G., Arciniega, A., Guzmán, M., Nava, E., Gutiérrez, C., Santoyo, M., 1995, Sismicidad del Volcán Popocatépetl a partir del 21 de Diciembre de 1994 al 30 de Marzo de 1995, en CENAPRED-UNAM(ed.), Volcán Popocatépetl, Estudios realizados durante la crisis de 1994-1995: México, D.F., 129-138.

Manuscrito recibido: Diciembre 5, 2006

Manuscrito corregido recibido: Febrero 2, 2007

Manuscrito aceptado: Febrero 9, 2007 